\title{
Super- and Anti-Principal-Modes in Multimode Waveguides
}

\author{
Philipp Ambichl, ${ }^{1}$ Wen Xiong, ${ }^{2}$ Yaron Bromberg, ${ }^{2}$ Brandon Redding, ${ }^{2}$ Hui Cao, ${ }^{2, *}$ and Stefan Rotter ${ }^{1, \dagger}$ \\ ${ }^{1}$ Institute for Theoretical Physics, Vienna University of Technology (TU Wien), \\ A-1040 Vienna, Austria, EU \\ ${ }^{2}$ Department of Applied Physics, Yale University, New Haven, Connecticut 06520, USA \\ (Received 7 April 2017; revised manuscript received 4 October 2017; published 30 November 2017)
}

\begin{abstract}
We introduce special states for light in multimode waveguides featuring strongly enhanced or reduced spectral correlations in the presence of strong mode coupling. Based on the experimentally measured multispectral transmission matrix of a multimode fiber, we generate a set of states that outperform the established "principal modes" in terms of the spectral stability of their output spatial field profiles. Inverting this concept also allows us to create states with a minimal spectral correlation width, whose output profiles are considerably more sensitive to a frequency change than typical input wave fronts. The resulting "superprincipal-modes" and "anti-principal-modes" are made orthogonal to each other even in the presence of mode-dependent loss. By decomposing them in the principal-mode basis, we show that the super-principalmodes are formed via interference of principal modes with close delay times, whereas the anti-principalmodes are a superposition of principal modes with the most-different delay times available in the fiber. Such novel states are expected to have broad applications in fiber communication, imaging, and spectroscopy.

DOI: 10.1103/PhysRevX.7.041053

Subject Areas: Complex Systems, Mesoscopics, Photonics
\end{abstract}

\section{INTRODUCTION}

Multimode optical fibers (MMFs) are complex and versatile systems that have wide-range applications from optical transmission [1-11], imaging [12-23], and manipulation [24,25] to high-power lasers [26,27] and amplifiers $[28,29]$. In all of these different contexts, the ability of MMFs to guide multiple transverse modes constitutes both a key feature and a considerable challenge. On the one hand, the availability of multiple modes dramatically increases the information transmission capacity of MMFs as compared to single-mode fibers (SMFs) carrying only a unique transverse mode. The large core of a MMF and the spread of optical energy over many spatial modes greatly weaken various nonlinear effects, enabling high-power operation for fiber-based lasers and amplifiers. On the other hand, the modal dispersion that comes with the different propagation constants of the fiber modes, together with the unavoidable random mixing between the modes, leads to severe spatial and temporal distortions of optical signals after propagating through the MMF.

One way to compensate for these distortions is provided by the eigenstates of the Wigner-Smith time-delay operator [30-42], also called the "principal modes" (PMs), which

\footnotetext{
*hui.cao@yale.edu

stefan.rotter@tuwien.ac.at
}

Published by the American Physical Society under the terms of the Creative Commons Attribution 4.0 International license. Further distribution of this work must maintain attribution to the author(s) and the published article's title, journal citation, and DOI. were introduced to suppress modal dispersion, allowing optical pulses with a sufficiently narrow spectral width to be sent through a MMF without distortion.

In addition, the PMs maintain the spatial coherence so that the output field can be focused to a diffractionlimited spot or converted to any spatial pattern $[39,40]$. The ability of maintaining temporal pulse shape and spatial coherence of a pulse amid strong spatiotemporal scattering is, in fact, desirable for many applications including fiber communication, endoscopy, and light amplification in MMFs [43-48]. Moreover, the PMs have great potential for quantum information protocols, as they can be used to synthesize decoherence-free, high-dimensional quantum states [49-51].

While PMs have already been studied theoretically in MMFs for more than ten years [32,52], creating them experimentally was only successful very recently in the weak $[39,42]$ and strong-mode-coupling regimes $[40,41]$. This is due to the fact that PMs require a carefully adjusted spatial input profile, both in amplitude and phase. Creating such tailored light waves has recently become possible because of advances in the development of spatial light modulators and the understanding of wave-front shaping techniques [53-60]. Since the concept of PMs is very general, it was realized that PMs should also exist in complex scattering systems (such as resonant cavities and disordered media) and for different types of waves (light, microwaves, sound, matter waves, etc.) [36]. In these new contexts, PMs also show characteristic features typically not present in MMFs, such as a particlelike wave profile that has also been observed both with acoustic waves [61] and with microwaves [62]. Furthermore, the 
concept of PMs can be generalized to realize wave fronts that focus on a designated target deep inside a disordered medium [63].

In spite of their promising potential, PMs suffer from two major shortcomings. First, they have a finite spectral width, limiting the bandwidth of input signals that can maintain the temporal pulse shape and spatial coherence after being transmitted. This limitation is severe for MMFs with strong mode mixing, as well as for disordered media, where the bandwidth of PMs is particularly narrow. Second, PMs are nonorthogonal to each other in non-Hermitian systems like a MMF with mode-dependent loss (MDL), resulting in crosstalk between them. A fundamental question is thus whether an orthogonal set of states exists that outperforms PMs in terms of the spectral correlation width, especially in the restrictive regime of strong mode coupling where a gain in bandwidth is in the highest demand. If such special states indeed exist, a practical question is how to generate them experimentally. Such an operational procedure could have a broad impact on all of the different complex wave-scattering systems where PMs can be generated, specifically for photonic applications in communication, imaging, nonlinear microscopy, laser amplifiers, and quantum technology.

In a seemingly unrelated context, it has recently been shown that a disordered medium [64] or a MMF [65-72] can function as a spectrometer with ultrahigh resolution, broad bandwidth, and low loss. The output speckle pattern of these systems is formed by multipath interference and thus changes with input frequency. The working principle of these spectrometers is to use the frequency-dependent speckle pattern as a fingerprint to recover the input spectrum. To further increase the resolving power of these devices, one needs to enhance the frequency sensitivity of output speckle patterns. The key question here is whether it is possible to achieve this by creating a special input state whose output-field pattern is considerably more sensitive to a frequency change than that of a typical input. The crucial point is, in other words, whether one can use the spatial degree of freedom at the input to accelerate the spectral decorrelation at the output of a disordered medium or of a MMF.

Another practical application of MMFs in biomedical imaging is to reduce the spatial coherence of a broadband light source for parallel optical coherence tomography (OCT) [73]. When imaging through turbid media such as biological tissue, the suppression of spatial coherence of the illuminating light prevents resolution loss from crosstalk due to coherent multiple scattering. If it was possible to create a state in the MMF with much reduced spectral correlation, one could greatly suppress the spatial coherence at the MMF output by launching broadband light into such a state.

Here, we introduce such novel states of light that have the aforementioned unique characteristics, and we generate them experimentally by addressing the spatial degrees of freedom of a MMF with wave-front shaping techniques. As we demonstrate explicitly, exceeding the already-efficient performance of PMs is possible by making use of the information stored in the multispectral transmission matrix of a MMF. Based on an optimization procedure operating on such a matrix [74], we are able to create a set of "superPMs" that not only have a significantly increased spectral stability as compared to the most stable PM available in the same fiber but that also have the great advantage of being mutually orthogonal even in the presence of MDL. Moreover, our optimization procedure can be applied to generate "anti-PMs" featuring an extremely narrow spectral correlation width - a property that holds promise for spectroscopy and sensing applications with MMFs.

Intuitively, the physical mechanisms for the formation of both super-PMs and anti-PMs can be understood in the basis of PMs. In the absence of MDL, super-PMs are formed by PMs with similar delay times, and the interference effect between them brings about a broader bandwidth for the super-PMs. On the contrary, anti-PMs are composed of PMs with extremely different delay times, which directly results in a bandwidth even narrower than that of random inputs. More importantly, our analysis illustrates that the PMs provide a powerful basis for synthesizing new types of states with unique spatial, temporal, and spectral characteristics.

\section{PRINCIPAL MODES}

Principal modes $\hat{\phi}$ are the eigenstates of the WignerSmith time-delay operator $q$ for a MMF with transmission matrix $t(\omega)[32,39,40,75]$,

$$
q \hat{\phi}=-\left.i t^{-1}\left(\omega_{0}\right) \frac{d t}{d \omega}\right|_{\omega=\omega_{0}} \hat{\phi}=\tau \hat{\phi},
$$

where $\tau$ is the respective complex eigenvalue whose real part is the so-called proper delay time (see Sec. V.C.1 in Ref. [60] for a review). From the above construction, it directly follows that PMs do not suffer from modal dispersion to the first order in frequency change (see Ref. [75]), such that the output-field pattern does not vary with frequency in the vicinity of $\omega_{0}$. A suitable measure for the modal dispersion, i.e., the deviation of the output vector $\vec{\psi}(\omega)=t(\omega) \hat{\phi}$ from its respective direction at $\omega_{0}$, is the field autocorrelation function $C\left(\omega, \omega_{0}\right):=\left|\hat{\psi}^{\dagger}(\omega) \hat{\psi}\left(\omega_{0}\right)\right| \in[0,1]$, where $\hat{\psi}(\omega)=\vec{\psi}(\omega) /|\vec{\psi}(\omega)|$ is the normalized output vector. We define the correlation width of a given input state as the spectral range surrounding $\omega_{0}$ where $C\left(\omega, \omega_{0}\right) \geq 0.9$ [40].

Experimentally, we tested a one-meter-long step-index MMF with a core diameter of $50 \mu \mathrm{m}$ and a numerical aperture of 0.22 . It features about 120 linearly polarized (LP) modes at $\lambda_{0}=1550 \mathrm{~nm}$ for one polarization. To directly access the regime of strong mode coupling, we 
apply stress to the fiber through external clamps. A tunable laser is employed for measuring the transmission matrix at different frequencies, with an incremental wavelength step of $0.01 \mathrm{~nm}$. Figure 1(a) shows our off-axis holographic setup for measuring the light field transmitted through the MMF. We use a spatial light modulator (SLM), which is imaged to the input facet of the MMF, to scan the incident angle of monochromatic laser light. For each input wave
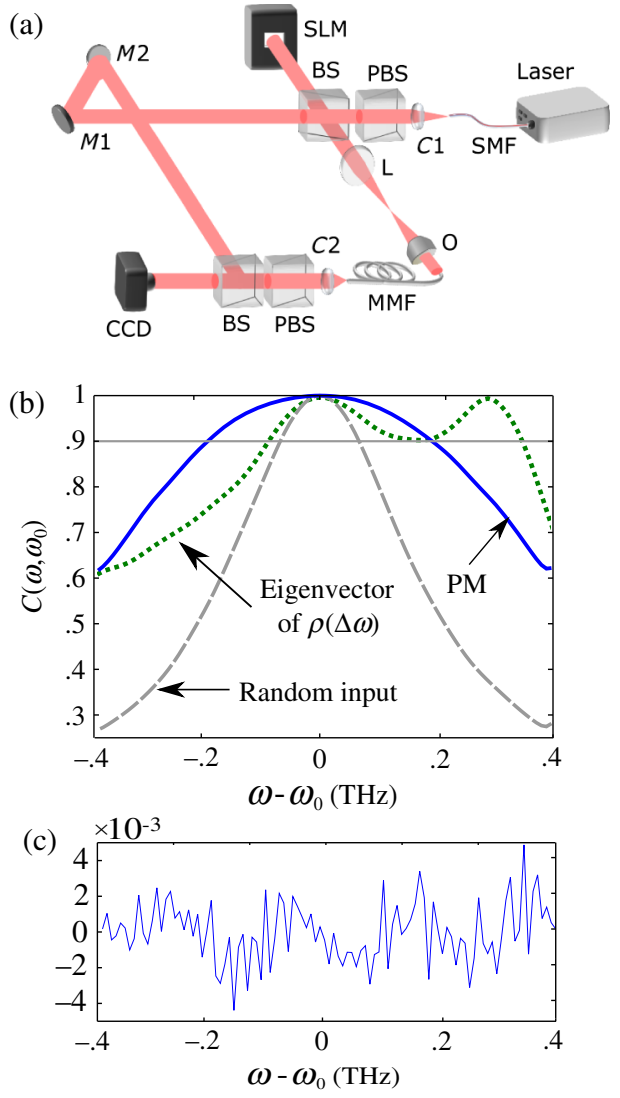

FIG. 1. (a) Schematics of the experimental setup. The output from a frequency-tunable laser, injected by a SMF, is collimated by a lens $(C 1)$ and linearly polarized by a polarization beam splitter (PBS) before being split by a beam splitter (BS) into two paths. (i) The signal is spatially modulated by the SLM and then imaged onto the fiber facet of the MMF by a lens $(L)$ and a microobjective $(O)$. (ii) The reference arm matches the signal arm's path length by a pair of mirrors $M_{1}$ and $M_{2}$. The output from the MMF is collimated $(C 2)$ and polarized (PBS) before combining with the reference signal at the BS with a relative angle. The interference fringes are recorded by a CCD camera. (b) Experimentally measured autocorrelation function $C\left(\omega, \omega_{0}\right)$ for the most stable PM (blue solid line). For comparison, we also show the measured average correlation of 20 random inputs (gray dashed line) with a narrower bandwidth. A super-PM eigenvector of $\rho(\Delta \omega)$ with $\Delta \omega=0.28 \mathrm{THz}$ is evaluated using the measured transmission matrix and its correlation function (green dotted curve). The horizontal solid gray line indicates the threshold value $C\left(\omega, \omega_{0}\right)=0.90$ that sets the correlation width. In panel (c), we plot the fluctuations of the measured correlation function of the PM shown in panel (b). front, the output signal from the fiber combines with the reference arm, forming interference fringes on the camera. We extract the amplitude and phase of the field from the interference pattern.

After recording the multispectral transmission matrix $t(\omega)$, we truncated the matrix to reduce noise by discarding the eigenchannels with very high loss. Out of 120 transmission channels, we discarded 30 that have transmission eigenvalues less than $1 \%$ of the largest eigenvalue. This threshold corresponds approximately to the experimental noise level in the measurement.

Based on the truncated transmission matrix, we compute the time-delay matrix $q$ and find its eigenstates (PMs). Applying a computer-generated phase hologram to the SLM, we create the PMs by constructing their input states with amplitude and phase modulations [76,77]. The outputfield patterns are recorded as the input frequency is scanned. Figure 1(b) shows the measured autocorrelation function $C\left(\omega, \omega_{0}\right)$ for the PM with the largest correlation width (blue solid line), which has about 3 times the width as compared to that of a random input wave front (gray dashed line). The experimental noise of the measured correlation function is smoothed out by applying a low-pass filter on the Fourier transform of the correlation function [the fluctuations suppressed in this way are shown in Fig. 1(c)].

\section{SUPER-PRINCIPAL-MODES}

The fundamental question we address here is whether it is possible to increase the correlation width beyond the values obtained for PMs. To achieve this goal, we revisit the design principle of PMs, which are generated by the operator $q$ in Eq. (1) such that their output-field patterns do not change when changing the input frequency incrementally by $d \omega$ from the reference value $\omega_{0}$ (see Ref. [75]). In a first step, we modify this approach to produce a state whose output pattern stays invariant when the input frequency is changed by a finite shift $\Delta \omega$ rather than by the infinitesimal value $d \omega$.

To implement such a strategy, we introduce a new operator that aligns the output vectors of its eigenstate at two arbitrarily spaced frequencies $\omega_{0}$ and $\omega$ (with $\left.\omega-\omega_{0}=\Delta \omega\right)$. This new operator $\rho\left(\omega, \omega_{0}\right)$ takes the following form:

$$
\rho\left(\omega, \omega_{0}\right):=-i t^{-1}\left(\omega_{0}\right) \frac{t(\omega)-t\left(\omega_{0}\right)}{\omega-\omega_{0}} .
$$

It approaches the time-delay operator $q$ for small frequency spacing between $\omega_{0}$ and $\omega$, i.e., $q=\lim _{\Delta \omega \rightarrow 0} \rho\left(\omega, \omega_{0}\right)$. An eigenstate of this new operator $\rho\left(\omega, \omega_{0}\right)$ features an output correlation function $C\left(\omega, \omega_{0}\right)$, which peaks not only at $\omega_{0}$ but also at $\omega$. With a gradual increase of $|\Delta \omega|$, the two correlation peaks move further apart, suggesting the possibility of extending the correlation bandwidth beyond that of the PM. We thereby use the term super-PM to label a state 
that has a correlation bandwidth of the output-field pattern exceeding that of the widest $\mathrm{PM}$ in a given fiber.

From the experimentally measured transmission matrix $t(\omega)$, we numerically generate the super-PMs as the eigenstates of $\rho\left(\omega, \omega_{0}\right)$ in Eq. (2) and find the anticipated two peaks at $\omega$ and $\omega_{0}$ in the correlation function, as shown by the green dotted curve in Fig. 1(b). The width of each peak is given approximately by the spectral correlation width $\delta \omega$ of the transmission matrix (i.e., the correlation width associated with random input). As the frequency spacing between the two peaks at $\omega$ and $\omega_{0}$ exceeds the peak width $\delta \omega$, a dip develops in between the two peaks. To maximize the correlation bandwidth, we choose the spacing $\omega-\omega_{0}$ such that $C\left(\omega, \omega_{0}\right)$ drops to the threshold value of 0.9 in between the two peaks in Fig. 1(b). In this case, the correlation width is about $18 \%$ wider than the width of the widest PM. A further increase of $\Delta \omega$ makes the dip in between the two peaks drop to below 0.9, causing a sudden decrease of bandwidth.

To reach the ideal case of a flat correlation function $C\left(\omega, \omega_{0}\right) \approx 1$ over the entire interval $\left[\omega_{0}, \omega\right], \Delta \omega$ must be less than $\delta \omega$. The fundamental reason that such a flat correlation curve is prevented from being realized in a broader frequency interval can be understood from the construction principle of $\rho\left(\omega, \omega_{0}\right)$ and of its eigenstates: A state with a flat correlation function $C\left(\omega, \omega_{0}\right) \equiv 1$ in a finite interval $\left[\omega_{0}, \omega\right]$ would have to be a simultaneous eigenstate of all operators $\rho\left(\omega, \omega_{0}\right)$ in this $\omega$ interval. The relevant commutator that would have to vanish for this to be possible is, however, nonzero in general: $\left[\rho\left(\omega_{1}, \omega_{0}\right), \rho\left(\omega_{2}, \omega_{0}\right)\right] \neq 0$ with $\left|\omega_{1}-\omega_{2}\right|>\delta \omega$.

In spite of this restriction, we now show how to create a set of states with correlation bandwidth considerably exceeding that of conventional PMs. In addition, we make such super-PMs mutually orthogonal, even when the fiber has MDL. Both the PMs and super-PMs described above are nonorthgonal, as the operators $q\left(\omega_{0}\right)$ and $\rho\left(\omega, \omega_{0}\right)$ become non-Hermitian in the presence of MDL. We thus work with a new approach and construct the desired set of super-PMs as approximate rather than as perfect simultaneous eigenvectors of the operators $\rho\left(\omega, \omega_{0}\right)$ within a desired spectral interval. Achieving this objective calls for the implementation of an optimization procedure with a nonlinear cost function involving the multispectral transmission matrix $t(\omega)$ and the operators $\rho\left(\omega, \omega_{0}\right)$. Given the dimensionality of the problem determined by the number of fiber modes and the width of the spectral region of interest, such an optimization is a very demanding task even numerically.

In the following, we take a shortcut to create orthogonal super-PMs by working with a considerably reduced optimization functional $\mathcal{T}$ (cost function),

$$
\begin{aligned}
\mathcal{T}(\hat{\phi}) & :=\int d \omega\left[1-C\left(\omega, \omega_{0}\right)^{2}\right] W(\omega) \\
& =\int d \omega\left[1-\frac{\left|\vec{\psi}^{\dagger}(\omega) \cdot \vec{\psi}\left(\omega_{0}\right)\right|^{2}}{|\vec{\psi}(\omega)|^{2}\left|\vec{\psi}\left(\omega_{0}\right)\right|^{2}}\right] W(\omega) .
\end{aligned}
$$

The spectral range that is effectively taken into account in this functional is defined by the weighting function $W(\omega)$, which is chosen to be a steplike but continuous function centered around $\omega_{0}$ [see Fig. 2(a) (purple dashdotted curve)]. To maximize the width of the correlation function $C\left(\omega, \omega_{0}\right)$, the (scalar) value of $\mathcal{T}$ needs to be minimized depending on the input state $\hat{\phi}=t^{-1}(\omega) \vec{\psi}(\omega)$. We implement an efficient minimization of $\mathcal{T}$ using a simple gradient-based scheme [see Ref. [75] for the exact expressions of $W(\omega)$ and $\delta \mathcal{T} / \delta \hat{\phi}]$. We note that our optimization scheme is a purely numerical optimization based on the experimentally measured transmission matrices rather than an experimental feedback loop.

We typically obtain the widest super-PM with an optimization loop that is initiated with the widest PM as (a)

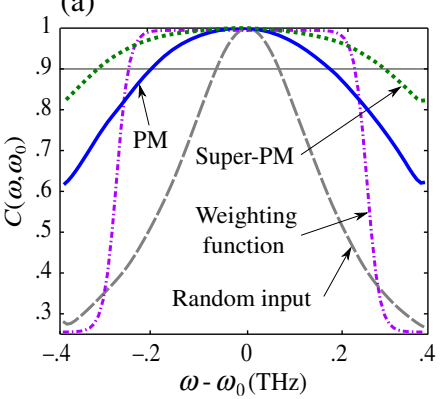

(b)

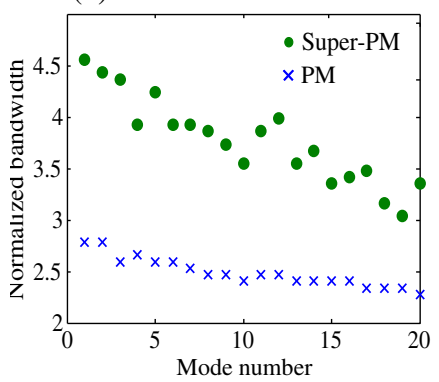

(c)

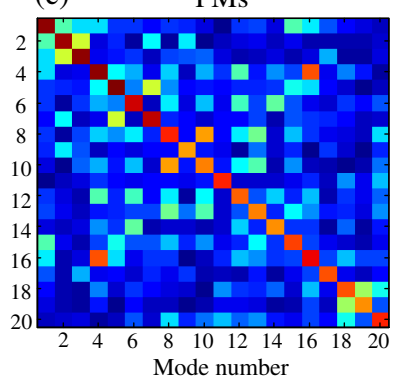

(d) Super-PMs

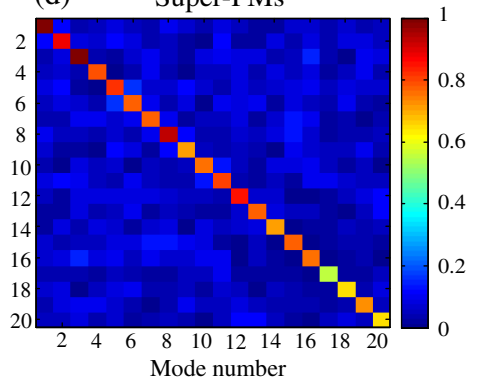

FIG. 2. (a) Measured spectral correlation function $C\left(\omega, \omega_{0}\right)$ for the output signals of the widest PM (blue solid line) and for the widest super-PM obtained from our bandwidth optimization (green dotted line). The weighting function $W(\omega)$ used in the cost function of Eq. (3) is shown as a purple dash-dotted line. The gray dashed line shows the spectral correlation of a random input. The horizontal solid gray line indicates the threshold value $C\left(\omega, \omega_{0}\right)=0.90$ that sets the correlation width. (b) Spectral correlation width of super-PMs and PMs normalized by that of a random input. Our optimization scheme found 20 super-PMs that have a larger bandwidth than the widest PM. The orthogonality of the output-field patterns for the 20 widest PMs (c) and the 20 super-PMs (d). In the presence of loss, the PMs are not orthogonal but the super-PMs are. 
a starting point for the corresponding iteration. Next, a whole cascade of optimizations of Eq. (3) is carried out, where in each optimization loop, for a single super-PM, the cost function $\mathcal{T}$ is minimized in the vector space orthogonal to each of the super-PMs obtained in the preceding steps. In this way, a strictly orthogonal set of super-PMs is obtained.

The input states for these super-PMs are created by the SLM and injected into the fiber. Figure 2(a) shows the widest super-PM (green dotted line). Its spectral correlation width is about $70 \%$ larger than that of the widest PM. Out of the total 90 modes in the fiber after the matrix truncation, we find 20 super-PMs that outperform the widest PM in terms of the correlation width. Figure 2(b) shows the spectral width of super-PMs normalized by that of random input wave fronts. The widest super-PM has a bandwidth increase of 4.5 times as compared to the random input. In addition, we also plot the 20 PMs with the largest bandwidth and find that the bandwidth enhancement for super-PMs is well above that of the PMs.

Because of fiber absorption and scattering loss, the PMs are nonorthogonal already at the fiber input since they are the eigenstates of the non-Hermitian operator $q$. The propagation through the fiber will further increase this nonorthogonality; thus, PMs are nonorthogonal at both the fiber input and output ends. By contrast, the super-PMs, in the way we construct them, are orthogonal at the input. We also observe that the orthogonality of the super-PMs at the input facet is well sustained during propagation through the fiber. The overlap of the output-field patterns is defined as $O_{m n}:=\left|\hat{\psi}_{m}^{\dagger}\left(\omega_{0}\right) \cdot \hat{\psi}_{n}\left(\omega_{0}\right)\right|$ for the $m$ th and $n$th super-PMs. For a perfectly orthonormal set of vectors, we would have $O_{m n}=\delta_{m n}$, where $\delta_{m n}$ is the Kronecker delta. The 20 widest PMs we consider are rather far away from this ideal case, as shown in Fig. 2(c). The average off-diagonal element of $O$ is 0.188 . The super-PMs preserve their initial orthogonality to a large extent, as shown in Fig. 2(d). On average, we find, for the off-diagonal elements of $O$, a reduced value of 0.068 . Thus, the crosstalk between different super-PMs is strongly suppressed as compared to the PMs.

To validate our initial hypothesis that the enhanced bandwidth of super-PMs is linked to the property that they mimic mutual eigenstates of all $\rho\left(\omega, \omega_{0}\right)$ operators in the spectral region of interest, we work out a corresponding measure that quantifies how close an input state is to a simultaneous eigenvector of these operators (see Ref. [75] for details). Applying this measure to the PMs and to the super-PMs indeed shows that the super-PMs are much closer to mutual eigenstates of $\rho\left(\omega, \omega_{0}\right)$ than the conventional PMs, despite the fact that the information on $\rho\left(\omega, \omega_{0}\right)$ is included in the cost function $\mathcal{T}$ only implicitly (see Ref. [75] for the corresponding numerical values we obtained).

Can we also understand, on a more intuitive level, why and how super-PMs manage to outperform PMs? To answer this question, we first decompose both the PMs and the super-PMs realized in the experiment in the basis of LP modes of the fiber. In the regime of strong mode coupling where all of the results shown above were obtained, we find that both PMs and super-PMs consist of nearly all LP modes, and the higher-order LP modes have smaller contributions due to higher loss (see Ref. [75]). While this finding confirms that PMs as well as super-PMs are really nontrivial combinations of fiber modes in the strong-coupling regime [40], it does not shed any light on the difference between PMs and super-PMs. We therefore change basis from the LP modes to the PMs themselves, which constitutes the natural basis to capture the dynamical aspects of light scattering as each PM is associated with a proper delay time. In addition, their greatly reduced wavelength dependence makes PMs very suitable to describe the transmitted light in a broad frequency window. When decomposing the super-PMs in the basis of PMs (with the biorthogonal basis vectors being sorted according to the delay times of the PMs), we clearly observe that the super-PMs are composed of PMs with neighboring delay times, as shown in Fig. 3(a).

The physical interpretation of this result is that superPMs build on a very narrow distribution of delay times and enhance the spectral correlation width via interference of several time-delay eigenstates. To demonstrate that the relative phases of constituent PMs are essential, in Fig. 3(b), we also plot the correlation functions after adding random phases to the decomposition coefficients of the PMs (while maintaining the absolute magnitudes). It is evident that randomizing the phase significantly decreases the correlation width. The sensitivity to the relative phase of constituent PMs indicates that the interference of the PMs involved in the formation of a super-PM is essential.

Another relevant aspect is that the mixing of neighboring PMs to create super-PMs requires that a sufficient number of PMs are available with similar delay times. In a MMF
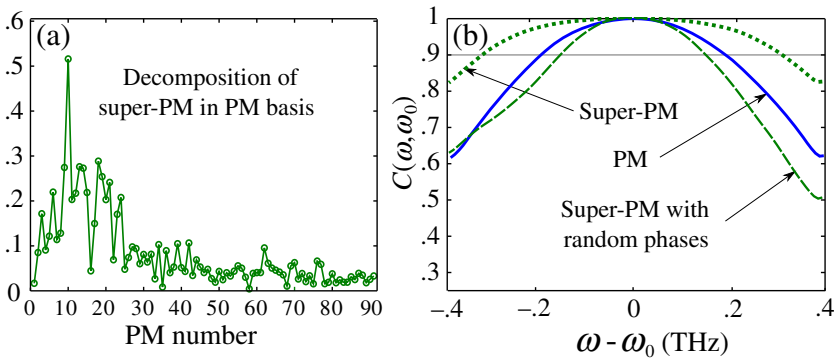

FIG. 3. (a) Decomposition of the widest super-PM in the basis of PMs, which are numbered from short to long delay times. The super-PM bundles together PMs with similar delay times. (b) Spectral correlation functions of the widest super-PM (green dotted line), the widest PM (blue solid line), and the widest superPM with phases of constituent PMs randomized (green dashed line). The bandwidth enhancement of super-PMs is sensitive to the phases of constituent PMs, indicating that super-PMs are formed by interference of PMs. 
with weak mode coupling, the values of the delay times are spread over a much broader time range; hence, the superPM is composed of only one or two PMs with neighboring delay times. Therefore, the bandwidth of a super-PM is typically not significantly different from its constituent PMs for weak mode coupling. To test this conjecture, we apply the same optimization algorithm as above to fibers in the weak-mode-coupling regime. The corresponding results indicate that, in this case, the super-PMs are very similar to individual PMs and no significant admixture from several PMs is seen. Consequently, the correlation width of even the widest super-PM barely exceeds that of the widest PM (see Ref. [75]). Super-PMs thus realize their full potential in the regime of strong mode coupling where a gain in bandwidth is also most relevant since the PM bandwidths in the strongcoupling limit are much narrower than for weak mode coupling [41].

\section{ANTI-PRINCIPAL-MODES}

We now investigate whether special states of light may also be found when turning the concept of principal modes on its head. Specifically, we address the question of whether it is possible to create not only states with a very broad spectral correlation width, such as the PMs or the super-PMs studied above, but also states with a drastically reduced bandwidth as compared to the values associated with a typical or random input wave front. Such anti-PMs would have an enhanced frequency sensitivity as desired, e.g., for fiber-based spectrometers whose operation principle relies on a very narrow correlation bandwidth $[65,66]$. The optimization algorithm presented above now allows us to generate such highly sensitive states by just maximizing instead of minimizing the functional $\mathcal{T}$ in Eq. (3).

We first apply the algorithm for anti-PMs on an ideal MMF with no MDL. The corresponding unitary transmission matrix is obtained numerically with the concatenated fiber model developed earlier [35]. For simplicity, we consider a planar waveguide with a core width of $200 \mu \mathrm{m}$ and a numerical aperture of 0.22 , supporting 57 guided modes [78]. The total length of the waveguide is 1 meter, divided into 20 segments, in each of which light propagates without mode coupling. Between adjacent segments, all modes are randomly coupled, as simulated by a unitary random matrix [41]. The results obtained by applying the optimization algorithm to this numerical model are shown in Fig. 4(a), displaying a significant reduction of the spectral correlation width of the anti-PM as compared to random inputs. Moreover, when decomposing the anti-PM in the basis of $\mathrm{PMs}$, we immediately see that the narrowest anti-PM consists mainly of admixtures between the fastest and slowest PMs [see Fig. 4(b)]. These PMs have the largest achievable temporal difference in the MMF, and mixing them with similar weight thus leads to an efficient decorrelation when changing the input frequency. Quite intuitively, the anti-PMs thus not only form the antipodes of the super-PMs in terms of
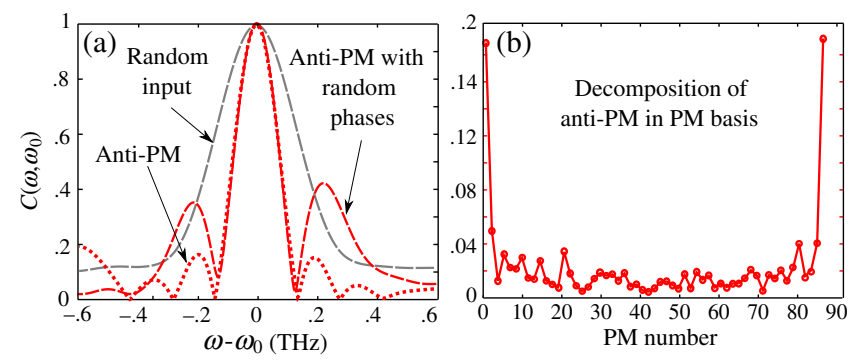

FIG. 4. Numerically determined anti-PM in a fiber without MDL. (a) Spectral correlation function of the narrowest anti-PM (red dotted line), which is significantly narrower than that of the random input (gray dashed line). The red dashed line is obtained by randomizing the phases of constituent PMs in the anti-PM. The phase randomization does not change the main peak, but it enhances the two side peaks. (b) The decomposition of this antiPM in the PM basis shows two pronounced maxima at the extreme values of the involved PMs, indicating that anti-PMs are efficiently formed by combining the fastest and the slowest PMs. The data shown here result from the numerical simulation of a waveguide with $200 \mu \mathrm{m}$ width, 0.22 numerical aperture, 1 meter length, and no loss.

their correlation bandwidth but also in terms of the way they are constructed: While super-PMs group together several PMs with similar time delays, the anti-PMs combine those PMs with the most-different time delays available. Whereas the relative phase with which this superposition of PMs is implemented clearly matters for super-PMs [see Fig. 3(b)], we find that for anti-PMs the correlation bandwidth is barely affected when changing the phases of constituent PMs [see the red dashed curve in Fig. 4(a)].

The reason behind this observation is that for PMs associated with very different delay times, adjusting their relative phase cannot mend these states' intrinsic tendency to decorrelate with frequency, while for states with nearby delay times, the appropriate phases will optimize the interferences for specific mode coupling in the MMF such as to increase the correlation width. As shown by the red dashed curve in Fig. 4(a), the correlation function features two enhanced side peaks adjacent to the main peak for the anti-PM with phases of constituent PMs randomized. After reaching zero, the correlation is revived as $\omega$ is tuned further from $\omega_{0}$. This revival is caused by the beating of the slowest and fastest PMs, the two main components of the anti-PM. When the relative phase of the fastest and slowest PMs accumulates $2 \pi$, the output field should be the same as the one when the relative phase is zero. However, small contributions from PMs with intermediate delay times give rise to multipath interference that suppresses the side peaks in the anti-PM in a way that is phase sensitive.

To connect with the experimental case of anti-PMs, it is essential to take into account the MDL in the fiber. We gradually increase the MDL in the numerical simulations up to the value close to the MMF used in the experiment. The narrowest anti-PM displays a narrowing of the spectral 

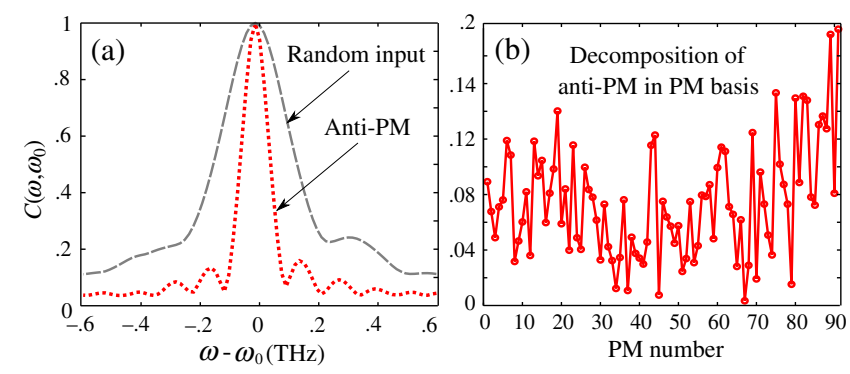

FIG. 5. Experimentally measured anti-PM in the same fiber as in Fig. 1. (a) The spectral correlation function of the narrowest anti-PM (red dotted line), which is notably narrower than that for a random input (gray dashed line). (b) Because of modedependent loss, the decomposition of this anti-PM in the PM basis does not give the bimodal distribution as in Fig. 4(b); instead, it gives a broad distribution over all available PMs. This result agrees with the numerical simulation that includes the MDL (see Ref. [75]).

correlation function, and its bimodal composition in the PM basis changes drastically (see Ref. [75]). In particular, the increase of MDL enhances the weight of the slowest PMs, and the composition of the anti-PM in the PM basis becomes much broader. This can be explained by considering that the MDL attenuates the slowest PMs most strongly since they stay inside the fiber the longest. The broadening of the PM composition can then be understood as a mechanism to compensate for the reduced contributions from the slow PMs. Since the constituent PMs with similar delay times overlap in time, their relative phases become important. When randomizing their phases, the bandwidth of anti-PMs is increased (see Ref. [75]).

When generating the anti-PMs in the MMF, we find 62 anti-PMs out of 90 fiber modes, whose spectral correlation width is narrower than that of random inputs. Figure 5 shows the data for the narrowest anti-PM. Because of the MDL, it is broadly spread in the PM basis, but it still decorrelates much more quickly than the random input with frequency detuning. Only when moving from the strong to the weak-modecoupling limit do we find that anti-PMs have about the same correlation width as a random input even without MDL (see Ref. [75]). This is because, in the weak-mode-coupling regime, the fastest PM and the slowest PM have very different delay times. The beating between the two PMs is so strong that it causes significant side peaks of the spectral correlation function. To reduce the side peaks, additional PMs with intermediate delay times must be included. The inclusion of many intermediate PMs makes the correlation width of the anti-PM similar to that of a random input. Therefore, both super-PMs and anti-PMs have in common that they unfold their full potential in the limit of strong mode coupling.

\section{SUMMARY}

In summary, we present a special set of light states in multimode fibers that have either a significantly broader spectral correlation than the principal modes or a significantly narrower spectral correlation than a random input wave front, respectively. We thus term these special states super-principal-modes and anti-principal-modes, and demonstrate how to generate them with a simple gradient-based algorithm based on the experimentally measured multispectral transmission matrix. Our optimization algorithm allows us to determine a whole set of such super- or antiPMs, which are mutually orthogonal to each other even in the presence of mode-dependent loss and thus feature greatly reduced crosstalk. By overcoming the limitations of PMs, super-PMs outperform the PMs in terms of bandwidth and orthogonality. The performance gain in terms of spectral stability is the highest in the regime of strong mode coupling as for very long fibers, where an increase in bandwidth is also most sought after, especially for communication purposes. For imaging applications, the fibers typically have a large number of modes, and our approach to control spectral stability is still applicable, although the optimization problem will be harder to solve. However, there is considerable room for improving the optimization strategy to identify super- and anti-PMs, and we expect follow-up studies to propose more efficient and problem-specific optimizers that outperform the all-purpose gradient-descent routine used here. Another topic for further study is the sensitivity of super- and anti-PMs with respect to fiber bending [14].

Our work also provides a physical understanding of how these states are formed by decomposing them in the PM basis. Super-PMs tend to combine several PMs with nearby delay times in a superposition with optimized phases. AntiPMs, on the contrary, tend to combine PMs with the mostdifferent delay times available in the fiber. The presence of MDLs leads to modifications of these states, which are analyzed in detail.

On the one hand, the large bandwidth and mutual orthogonality of super-PMs pave the way for their application to dispersion-free transmission of pulses through complex media. On the other hand, the high spectral sensitivity of anti-PMs makes them ideally suited for optimizing the resolution of speckle spectrometers based on disordered media or on MMFs, as well as for reducing the spatial coherence of a broadband light source for crosstalkfree imaging [79]. Since neither the concept of super- or antiPMs nor the ability to create complex wave fields with wave-front shaping is restricted to a specific type of scattering system or to a specific type of wave, we expect our results to be easily transferable to other experimental platforms. In this context, we note that the original definition of the time-delay matrix for a multiple scattering medium relies on the scattering matrix, which includes both transmission and reflection. However, one can define an effective time-delay matrix for the transmitted light, and an eigenstate of this matrix, which involves only the transmission matrix, has a well-defined delay time for the 
transmitted light. Such a state makes the total transmission dispersion-free, in contrast to the spatial-temporal focusing, which achieves dispersion-free transmission only for a single spatial channel $[45,80,81]$. Therefore, we foresee no obstacle to transfer the concepts of PMs, super-PMs, and anti-PMs to disordered media with reflection and loss.

\section{ACKNOWLEDGMENTS}

We acknowledge Chia Wei Hsu, Joel Carpenter, and Nicolas Fontaine for helpful discussions. This work is supported partly by the U.S. National Science Foundation under Grant No. ECCS-1509361 and by the U.S. Office of Naval Research under MURI Grant No. N00014-13-10649. P. A. and S. R. acknowledge support by the Austrian Science Fund (FWF) through Projects SFB NextLite (F49-P10) and by the European Commission under Project NHQWAVE (Grant Agreement No. 691209).

[1] D. J. Richardson, J. M. Fini, and L. E. Nelson, SpaceDivision Multiplexing in Optical Fibres, Nat. Photonics 7, 354 (2013).

[2] R.-J. Essiambre, G. Kramer, P. J. Winzer, G. J. Foschini, and B. Goebel, Capacity Limits of Optical Fiber Networks, J. Lightwave Technol. 28, 662 (2010).

[3] J. M. Kahn and D. A. B. Miller, Communications Expands Its Space, Nat. Photonics 11, 5 (2017).

[4] S. Randel, R. Ryf, A. Sierra, P. J. Winzer, A. H. Gnauck, C. A. Bolle, R.-J. Essiambre, D. W. Peckham, A. McCurdy, and R. Lingle, $6 \times 56-\mathrm{gb} / \mathrm{s}$ Mode-Division Multiplexed Transmission over 33-km Few-Mode Fiber Enabled by 6×6 MIMO Equalization, Opt. Express 19, 16697 (2011).

[5] R. Ryf, S. Randel, A. H. Gnauck, C. Bolle, A. Sierra, S. Mumtaz, M. Esmaeelpour, E. C. Burrows, R.-J. Essiambre, P. J. Winzer et al., Mode-Division Multiplexing over $96 \mathrm{~km}$ of Few-Mode Fiber Using Coherent $6 \times 6$ MIMO Processing, J. Lightwave Technol. 30, 521 (2012).

[6] R. Ryf, S. Randel, A. H. Gnauck, C. Bolle, R.-J. Essiambre, P. Winzer, D. W. Peckham, A. McCurdy, and R. Lingle, Space-Division Multiplexing over $10 \mathrm{~km}$ of Three-Mode Fiber Using Coherent $6 \times 6$ MIMO Processing, in National Fiber Optic Engineers Conference (Optical Society of America, Washington, DC, 2011), p. PDPB10.

[7] R. Ryf, S. Randel, N. K. Fontaine, M. Montoliu, E. Burrows, S. Chandrasekhar, A. H. Gnauck, C. Xie, R.-J. Essiambre, P. Winzer et al., 32-bit/s/Hz Spectral Efficiency WDM Transmission over 177-km Few-Mode Fiber, in Optical Fiber Communication Conference (Optical Society of America, 2013), pp. PDP5A-1.

[8] N. K. Fontaine, R. Ryf, H. Chen, A. Velazquez Benitez, J. E. Antonio Lopez, R. Amezcua Correa, B. Guan, B. Ercan, R. P. Scott, S. J. Ben Yoo et al., $30 \times 30$ MIMO Transmission over 15 Spatial Modes, in Optical Fiber Communications Conference and Exhibition (IEEE, 2015), pp. 1-3.

[9] M. Salsi, C. Koebele, D. Sperti, P. Tran, P. Brindel, H. Mardoyan, S. Bigo, A. Boutin, F. Verluise, P. Sillard et al., Transmission at $2 \times 100 \mathrm{~Gb} / \mathrm{s}$, over Two Modes of
40 km-long Prototype Few-Mode Fiber, Using LCOS Based Mode Multiplexer and Demultiplexer, in Optical Fiber Communication Conference (Optical Society of America, 2011), p. PDPB9.

[10] J. Carpenter, B. C. Thomsen, and T. D. Wilkinson, Degenerate Mode-Group Division Multiplexing, J. Lightwave Technol. 30, 3946 (2012).

[11] J. Carpenter and T.D. Wilkinson, All Optical ModeMultiplexing Using Holography and Multimode Fiber Couplers, J. Lightwave Technol. 30, 1978 (2012).

[12] B. A. Flusberg, E. D. Cocker, W. Piyawattanametha, J. C. Jung, E. L. M. Cheung, and M. J. Schnitzer, Fiber-Optic Fluorescence Imaging, Nat. Methods 2, 941 (2005).

[13] Y. Choi, C. Yoon, M. Kim, T. D. Yang, C. Fang-Yen, R. R. Dasari, K. J. Lee, and W. Choi, Scanner-Free and Wide-Field Endoscopic Imaging by Using a Single Multimode Optical Fiber, Phys. Rev. Lett. 109, 203901 (2012).

[14] M. Plöschner, T. Tyc, and T. Čižmár, Seeing through Chaos in Multimode Fibres, Nat. Photonics 9, 529 (2015).

[15] T. Čižmár and K. Dholakia, Exploiting Multimode Waveguides for Pure Fibre-Based Imaging, Nat. Commun. 3, 1027 (2012).

[16] M. Plöschner and T. Čižmár, Compact Multimode Fiber Beam-Shaping System Based on GPU Accelerated Digital Holography, Opt. Lett. 40, 197 (2015).

[17] T. Čižmár and K. Dholakia, Shaping the Light Transmission through a Multimode Optical Fibre: Complex Transformation Analysis and Applications in Biophotonics, Opt. Express 19, 18871 (2011).

[18] L. V. Amitonova, A. Descloux, J. Petschulat, M. H. Frosz, G. Ahmed, F. Babic, X. Jiang, A. P. Mosk, P. St. J. Russell, and P. W. H. Pinkse, High-Resolution Wavefront Shaping with a Photonic Crystal Fiber for Multimode Fiber Imaging, Opt. Lett. 41, 497 (2016).

[19] I. N. Papadopoulos, S. Farahi, C. Moser, and D. Psaltis, High-Resolution, Lensless Endoscope Based on Digital Scanning through a Multimode Optical Fiber, Biomed. Opt. Express 4, 260 (2013).

[20] E. E. Morales-Delgado, D. Psaltis, and C. Moser, Two-Photon Imaging through a Multimode Fiber, Opt. Express 23, 32158 (2015).

[21] N. Stasio, C. Moser, and D. Psaltis, Calibration-Free Imaging through a Multicore Fiber Using Speckle Scanning Microscopy, Opt. Lett. 41, 3078 (2016).

[22] D. Loterie, D. Psaltis, and C. Moser, Bend Translation in Multimode Fiber Imaging, Opt. Express 25, 6263 (2017).

[23] S. M. Kolenderska, O. Katz, M. Fink, and S. Gigan, Scanning-Free Imaging through a Single Fiber by Random Spatio-Spectral Encoding, Opt. Lett. 40, 534 (2015).

[24] A. Maimaiti, V. G. Truong, M. Sergides, I. Gusachenko, and S. N. Chormaic, Higher Order Microfibre Modes for Dielectric Particle Trapping and Propulsion, Sci. Rep. 5, 9077 (2015).

[25] S. Bianchi and R. Di Leonardo, A Multi-mode Fiber Probe for Holographic Micromanipulation and Microscopy, Lab Chip 12, 635 (2012).

[26] H. Li, Modeling Compact High Power Fiber Lasers and VECSELs (University of Arizona, Tucson, AZ, 2011). 
[27] O. G. Okhotnikov, Fiber Lasers (John Wiley \& Sons, New York, 2012).

[28] N. Bai, E. Ip, T. Wang, and G. Li, Multimode Fiber Amplifier with Tunable Modal Gain Using a Reconfigurable Multimode Pump, Opt. Express 19, 16601 (2011).

[29] N. K. Dutta, Fiber Amplifiers and Fiber Lasers (World Scientific, Singapore, 2014).

[30] E. P. Wigner, Lower Limit for the Energy Derivative of the Scattering Phase Shift, Phys. Rev. 98, 145 (1955).

[31] F. T. Smith, Lifetime Matrix in Collision Theory, Phys. Rev. 118, 349 (1960).

[32] S. Fan and J. M. Kahn, Principal Modes in Multimode Waveguides, Opt. Lett. 30, 135 (2005).

[33] A. A. Juarez, C. A. Bunge, S. Warm, and K. Petermann, Perspectives of Principal Mode Transmission in ModeDivision-Multiplex Operation, Opt. Express 20, 13810 (2012).

[34] M. B. Shemirani, W. Mao, R. A. Panicker, and J. M. Kahn, Principal Modes in Graded-Index Multimode Fiber in Presence of Spatial- and Polarization-Mode Coupling, J. Lightwave Technol. 27, 1248 (2009).

[35] K. P. Ho and J. M. Kahn, Statistics of Group Delays in Multimode Fiber with Strong Mode Coupling, J. Lightwave Technol. 29, 3119 (2011).

[36] S. Rotter, P. Ambichl, and F. Libisch, Generating Particlelike Scattering States in Wave Transport, Phys. Rev. Lett. 106, 120602 (2011).

[37] B. Franz and H. Bulow, Experimental Evaluation of Principal Mode Groups as High-Speed Transmission Channels in Spatial Multiplex Systems, IEEE Photonics Technol. Lett. 24, 1363 (2012).

[38] G. Milione, D. A. Nolan, and R. R. Alfano, Determining Principal Modes in a Multimode Optical Fiber Using the Mode Dependent Signal Delay Method, J. Opt. Soc. Am. B 32, 143 (2015).

[39] J. Carpenter, B. J. Eggleton, and J. Schröder, Observation of Eisenbud-Wigner-Smith States as Principal Modes in Multimode Fibre, Nat. Photonics 9, 751 (2015).

[40] W. Xiong, P. Ambichl, Y. Bromberg, B. Redding, S. Rotter, and H. Cao, Spatiotemporal Control of Light Transmission through a Multimode Fiber with Strong Mode Coupling, Phys. Rev. Lett. 117, 053901 (2016).

[41] W. Xiong, P. Ambichl, Y. Bromberg, B. Redding, S. Rotter, and H. Cao, Principal Modes in Multimode Fibers: Exploring the Crossover from Weak to Strong Mode Coupling, Opt. Express 25, 2709 (2017).

[42] J. Carpenter, B. J. Eggleton, and J. Schröder, Comparison of Principal Modes and Spatial Eigenmodes in Multimode Optical Fibre, Laser Photonics Rev. 11, 1600259 (2017).

[43] M. Kim, Y. Choi, C. Yoon, W. Choi, J. Kim, Q.-H. Park, and W. Choi, Maximal Energy Transport through Disordered Media with the Implementation of Transmission Eigenchannels, Nat. Photonics 6, 581 (2012).

[44] I. N. Papadopoulos, S. Farahi, C. Moser, and D. Psaltis, Focusing and Scanning Light through a Multimode Optical Fiber Using Digital Phase Conjugation, Opt. Express 20, 10583 (2012).

[45] J. Aulbach, B. Gjonaj, P. M. Johnson, A. P. Mosk, and A. Lagendijk, Control of Light Transmission through Opaque
Scattering Media in Space and Time, Phys. Rev. Lett. 106, 103901 (2011).

[46] D. J. Richardson, J. Nilsson, and W. A. Clarkson, High Power Fiber Lasers: Current Status and Future Perspectives, J. Opt. Soc. Am. B 27, B63 (2010).

[47] C. Jauregui, J. Limpert, and A. Tünnermann, High-Power Fibre Lasers, Nat. Photonics 7, 861 (2013).

[48] R. Florentin, V. Kermene, J. Benoist, A. DesfargesBerthelemot, D. Pagnoux, A. Barthélémy, and J.-P. Huignard, Shaping the Light Amplified in a Multimode Fiber, Light Sci. Appl. 6, e16208 (2017).

[49] A. Peruzzo, A. Laing, A. Politi, T. Rudolph, and J. L. O'Brien, Multimode Quantum Interference of Photons in Multiport Integrated Devices, Nat. Commun. 2, 224 (2011).

[50] A. Peruzzo, M. Lobino, J. C. F. Matthews, N. Matsuda, A. Politi, K. Poulios, X.-Q. Zhou, Y. Lahini, N. Ismail, K. Wörhoff et al., Quantum Walks of Correlated Photons, Science 329, 1500 (2010).

[51] H. Defienne, M. Barbieri, I. A. Walmsley, B. J. Smith, and S. Gigan, Two-Photon Quantum Walk in a Multimode Fiber, Sci. Adv. 2, e1501054 (2016).

[52] X. Shen, J. M. Kahn, and M. A. Horowitz, Compensation for Multimode Fiber Dispersion by Adaptive Optics, Opt. Lett. 30, 2985 (2005).

[53] I. M. Vellekoop and A. P. Mosk, Focusing Coherent Light through Opaque Strongly Scattering Media, Opt. Lett. 32, 2309 (2007).

[54] I. M. Vellekoop, A. Lagendijk, and A. P. Mosk, Exploiting Disorder for Perfect Focusing, Nat. Photonics 4, 320 (2010).

[55] S. M. Popoff, G. Lerosey, R. Carminati, M. Fink, A. C. Boccara, and S. Gigan, Measuring the Transmission Matrix in Optics: An Approach to the Study and Control of Light Propagation in Disordered Media, Phys. Rev. Lett. 104, 100601 (2010).

[56] S. M. Popoff, G. Lerosey, M. Fink, A. C. Boccara, and S. Gigan, Controlling Light through Optical Disordered Media: Transmission Matrix Approach, New J. Phys. 13, 123021 (2011).

[57] S. M. Popoff, G. Lerosey, M. Fink, A. C. Boccara, and S. Gigan, Image Transmission through an Opaque Material, Nat. Commun. 1, 81 (2010).

[58] O. Katz, E. Small, Y. Bromberg, and Y. Silberberg, Focusing and Compression of Ultrashort Pulses through Scattering Media, Nat. Photonics 5, 372 (2011).

[59] A. P. Mosk, A. Lagendijk, G. Lerosey, and M. Fink, Controlling Waves in Space and Time for Imaging and Focusing in Complex Media, Nat. Photonics 6, 283 (2012).

[60] S. Rotter and S. Gigan, Light Fields in Complex Media: Mesoscopic Scattering Meets Wave Control, Rev. Mod. Phys. 89, 015005 (2017).

[61] B. Gérardin, J. Laurent, P. Ambichl, C. Prada, S. Rotter, and A. Aubry, Particlelike Wave Packets in Complex Scattering Systems, Phys. Rev. B 94, 014209 (2016).

[62] J. Böhm, A. Brandstötter, P. Ambichl, S. Rotter, and U. Kuhl, Particlelike Scattering States in a Microwave Cavity, arXiv:1706.08926.

[63] P. Ambichl, A. Brandstötter, J. Böhm, M. Kühmayer, U. Kuhl, and S. Rotter, Focusing inside Disordered Media with 
the Generalized Wigner-Smith Operator, Phys. Rev. Lett. 119, 033903 (2017).

[64] B. Redding, S. F. Liew, R. Sarma, and H. Cao, Compact Spectrometer Based on a Disordered Photonic Chip, Nat. Photonics 7, 746 (2013).

[65] B. Redding and H. Cao, Using a Multimode Fiber as a High-Resolution, Low-Loss Spectrometer, Opt. Lett. 37, 3384 (2012).

[66] B. Redding, S. M. Popoff, and H. Cao, All-Fiber Spectrometer Based on Speckle Pattern Reconstruction, Opt. Express 21, 6584 (2013).

[67] B. Redding, S. M. Popoff, Y. Bromberg, M. A. Choma, and H. Cao, Noise Analysis of Spectrometers Based on Speckle Pattern Reconstruction, Appl. Opt. 53, 410 (2014).

[68] B. Redding, M. Alam, M. Seifert, and H. Cao, HighResolution and Broadband All-Fiber Spectrometers, Optica 1, 175 (2014).

[69] B. Redding, S. F. Liew, Y. Bromberg, R. Sarma, and H. Cao, Evanescently Coupled Multimode Spiral Spectrometer, Optica 3, 956 (2016).

[70] S. F. Liew, B. Redding, M. A. Choma, H. D. Tagare, and H. Cao, Broadband Multimode Fiber Spectrometer, Opt. Lett. 41, 2029 (2016).

[71] N. Coluccelli, M. Cassinerio, B. Redding, H. Cao, P. Laporta, and G. Galzerano, The Optical Frequency Comb Fibre Spectrometer, Nat. Commun. 7, 12995 (2016).

[72] G. C. Valley, G. A. Sefler, and T. J. Shaw, Multimode Waveguide Speckle Patterns for Compressive Sensing, Opt. Lett. 41, 2529 (2016).
[73] A.-H. Dhalla, J. V. Migacz, and J. A. Izatt, Crosstalk Rejection in Parallel Optical Coherence Tomography Using Spatially Incoherent Illumination with Partially Coherent Sources, Opt. Lett. 35, 2305 (2010).

[74] D. Andreoli, G. Volpe, S. Popoff, O. Katz, S. Gresillon, and S. Gigan, Deterministic Control of Broadband Light through a Multiply Scattering Medium via the Multispectral Transmission Matrix, Sci. Rep. 5, 10347 (2015).

[75] See Supplemental Material at http://link.aps.org/ supplemental/10.1103/PhysRevX.7.041053 for detailed analytical calculations and additional figures.

[76] V. Arrizón, U. Ruiz, R. Carrada, and L. A. González, Pixelated Phase Computer Holograms for the Accurate Encoding of Scalar Complex Fields, J. Opt. Soc. Am. A 24, 3500 (2007).

[77] V. Bagnoud and J. D. Zuegel, Independent Phase and Amplitude Control of a Laser Beam by Use of a Single-PhaseOnly Spatial Light Modulator, Opt. Lett. 29, 295 (2004).

[78] K. Okamoto, Fundamentals of Optical Waveguides (Elsevier, New York, 2005).

[79] H. Cao, Perspective on Speckle Spectrometers, J. Opt. (India) 19, 060402 (2017).

[80] D. J. McCabe, A. Tajalli, D. R. Austin, P. Bondareff, I. A. Walmsley, S. Gigan, and B. Chatel, Spatio-Temporal Focusing of an Ultrafast Pulse through a Multiply Scattering Medium, Nat. Commun. 2, 447 (2011).

[81] O. Katz, E. Small, Y. Bromberg, and Y. Silberberg, Focusing and Compression of Ultrashort Pulses through Scattering Media, Nat. Photonics 5, 372 (2011). 